\title{
La durée de l'isolement conjugal et de la vie en couple chez les personnes âgées en France : quelles évolutions entre hommes et femmes au fil des générations ? \\ Time spent in unpartnered and partnered states among old people in France : What has changed for men and women over time?
}

\section{Sophie Pennec et Joëlle Gaymu}

Volume 40, numéro 2, automne 2011

Microsimulation en démographie

URI : https://id.erudit.org/iderudit/1011539ar

DOI : https://doi.org/10.7202/1011539ar

Aller au sommaire du numéro

Éditeur(s)

Association des démographes du Québec

ISSN

0380-1721 (imprimé)

1705-1495 (numérique)

Découvrir la revue

Citer cet article

Pennec, S. \& Gaymu, J. (2011). La durée de l'isolement conjugal et de la vie en couple chez les personnes âgées en France : quelles évolutions entre hommes et femmes au fil des générations ? Cahiers québécois de démographie, 40(2),

175-208. https://doi.org/10.7202/1011539ar

\section{Résumé de l'article}

L'objectif essentiel de cet article est de quantifier l'évolution de la durée de l'isolement matrimonial (années vécues dans le célibat, le veuvage ou le divorce), période durant laquelle les personnes âgées sont plus vulnérables surtout en cas de dépendance. Nos analyses s'appuient sur un modèle de microsimulation qui a permis d'évaluer l'évolution des durées de vie dans les différents états matrimoniaux pour les générations de personnes âgées de 60 ans et plus nées entre 1900 à 1950. Au fil des générations, les durées passées dans le mariage des hommes et des femmes se sont allongées et rapprochées. Toutefois, ce sont, pour l'essentiel, les générations passées et présentes d'aînés qui ont bénéficié de cette tendance. À partir de la génération 1930, le temps passé dans le mariage devrait davantage s'allonger chez les femmes que chez les hommes, l'inverse valant pour l'isolement matrimonial. 
Cahiers québécois de démographie

Vol. 40, $\mathrm{n}^{\circ}$ 2, automne 2011, p. 175-208

\title{
La durée de l'isolement conjugal et de la vie en couple chez les personnes âgées en France: quelles évolutions entre hommes et femmes au fil des générations?
}

\author{
SOPHIE PENNEC ${ }^{\star}$ ET JOËLLE GAYMU ${ }^{\star}$
}

\begin{abstract}
L'objectif essentiel de cet article est de quantifier l'évolution de la durée de l'isolement matrimonial (années vécues dans le célibat, le veuvage ou le divorce), période durant laquelle les personnes âgées sont plus vulnérables surtout en cas de dépendance. Nos analyses s'appuient sur un modèle de microsimulation qui a permis d'évaluer l'évolution des durées de vie dans les différents états matrimoniaux pour les générations de personnes âgées de 60 ans et plus nées entre 1900 à 1950. Au fil des générations, les durées passées dans le mariage des hommes et des femmes se sont allongées et rapprochées. Toutefois, ce sont, pour l'essentiel, les générations passées et présentes d'aînés qui ont bénéficié de cette tendance. À partir de la génération 1930, le temps passé dans le mariage devrait davantage s'allonger chez les femmes que chez les hommes, l'inverse valant pour l'isolement matrimonial.
\end{abstract}

English abstract, p. 208

\section{INTRODUCTION}

T e statut matrimonial est un facteur clé des conditions de vie des personnes âgées et par là même de leur qualité de vie. On sait, par exemple, que ceux qui vivent à deux sont dans une situation économique plus favorable, ont un meilleur état de santé (Glaser et collab., 1997) et sont mieux intégrés socialement (Delbès et Gaymu, 2003a; De Jong Gierveld et collab., 1997).

* Institut national d'études démographiques (INED), Paris, France (pennec@ined.fr; gaymu@ined.fr). Les auteurs tiennent à remercier les relecteurs anonymes pour leurs suggestions pertinentes ainsi que Rob Hyndman pour son aide dans l'utilisation de certains programmes $\mathrm{R}$. 
Or, résultat bien connu, hommes et femmes ont des risques très différents de vieillir en couple. Dans les générations actuelles de retraités, la surmortalité masculine, l'écart d'âge au mariage et les plus fortes chances de remariage des hommes conjuguent leurs effets dans le sens d'un très fort veuvage féminin, alors qu'à l'inverse les hommes restent généralement mariés jusqu'à leur décès : ainsi, en 2005 en France, plus des trois quarts des hommes de 60 ans ou plus sont mariés contre moins d'une femme sur $\operatorname{deux}(46 \%)$.

La situation matrimoniale a également une influence majeure sur la structure des ménages des personnes âgées : quasiment toutes celles qui sont mariées vivent en couple alors que veufs et divorcés, dont les modes de vie sont proches, habitent majoritairement seuls. C'est, du reste, essentiellement parce que les femmes sont plus fréquemment veuves qu'elles sont vouées à l'isolement résidentiel (Delbès, Gaymu et Springer, 2006 ; United Nations, 2005). Quant aux célibataires, ils se singularisent par leur plus forte tendance à opter pour l'institutionnalisation (Dolinsky et Rosenwaike, 1988; Grundy et Glaser, 1997; Ricci, 1991). Le fait qu'ils soient généralement sans proche parent y est pour beaucoup (Angel et Himes, 1992 ; Desesquelles et Brouard, 2003; Soldo et collab., 1990). On sait, en effet, qu'en cas de dépendance, conjoint et enfants sont les premiers pourvoyeurs d'aide (Chappell, 1991; Walker et collab., 1993) : l'absence ou la défaillance de ces soutiens augmente, ipso facto, le besoin d'aide formelle.

De plus, l'on sait que l'intensité et les formes du soutien familial sont fortement déterminées par le statut matrimonial tant des parents que des enfants. Par exemple, les divorcés, tout particulièrement côté masculin, viennent moins souvent en aide à leurs parents et reçoivent moins de soutien de la part de leurs enfants (Cooney et Uhlenberg, 1990 ; AttiasDonfut, 1995).

Ces considérations générales montrent donc à quel point la situation matrimoniale est une donnée fondamentale tant sur le plan individuel que collectif et à quel point aussi il modèle différemment la vieillesse des hommes et des femmes. Les grandes évolutions des structures matrimoniales des personnes âgées ont fait l'objet de multiples recherches. À chaque âge, le veuvage est en constante régression au cours du temps, en raison, essentiellement, du recul de la probabilité de décéder pour chacun des membres du couple. Demain, compte tenu de la probable poursuite de la baisse de la mortalité, l'âge de la rupture d'union par veuvage devrait se produire plus tardivement encore. Mais certaines études ont montré que, à l'avenir, la progression des divorces allait contrecarrer ces tendances et 
entraîner plus d'isolement matrimonial durant la vieillesse (Delbès et Gaymu, 2003b ; Kalogirou et Murphy, 2006; Wilson et Smallwood, 2008).

Par-delà ces constats d'une modification de la structure matrimoniale des personnes âgées, l'objectif de cet article est de quantifier l'évolution des durées passées dans chacun des états matrimoniaux. En effet, une baisse du risque de devenir veuf (lié à la probabilité de survivre en tant que marié et au risque de décéder de son conjoint) ne signifie pas nécessairement une diminution de la durée de veuvage (lié à la survie en tant que veuf). En outre, même si devenir veuf est de moins en moins fréquent à chaque âge, il n'en reste pas moins vrai qu'à l'avenir de nombreuses personnes âgées seront toujours confrontées à cet événement. Dans ce cas, on peut, notamment, se demander si cette période d'isolement matrimonial s'est allongée, au fil des générations, de façon identique pour les hommes et pour les femmes. Les mêmes interrogations se posent pour les divorcés. L'isolement conjugal ne signifie pas forcément isolement social, mais cette situation fragilise les individus, surtout en cas de dépendance. L'on sait, en effet, qu'au quotidien les couples arrivent mieux à assumer les tâches domestiques du fait du partage et de la spécialisation des rôles : ainsi, en France, seuls $9 \%$ des 80 ans ou plus vivant à deux ne font jamais leurs courses contre $36 \%$ des veuves du même âge (David et Starzec, 1996). Les personnes âgées dépendantes vivant seules ont plus souvent recours à de l'aide formelle que celles vivant en couple ou avec d'autres personnes (Arber et collab., 1988; Grundy, 2006; Pickard et collab., 200o). En France, par exemple, tel est le cas des deux tiers des personnes âgées dépendantes vivant seules contre la moitié de celles vivant en couple (Breuil-Genier, 1998). Dans ce cas, la durée de la prise en charge a des conséquences majeures en termes de coût collectif mais aussi familial : les aidants familiaux, essentiellement les femmes, y font généralement face, au détriment de leur propre santé (Schulz et collab., 1995) et au prix d'aménagements, parfois importants, de leur carrière professionnelle (Ettner, 1995; Spiess et Schneider, 2003).

Il s'agit donc ici de quantifier l'évolution de la durée de l'isolement matrimonial (années vécues dans le célibat, le veuvage ou le divorce), période durant laquelle les personnes âgées sont plus vulnérables surtout en cas de dépendance. On s'attachera, en particulier, à prendre en compte une variété de parcours matrimoniaux. En effet, certains vivront avec ou sans partenaire jusqu'à leur décès alors que d'autres connaîtront des périodes successives de vie en couple et en solitaire à la suite d'un veuvage ou un divorce, ces ruptures pouvant intervenir plus ou moins précocement dans le cycle de vie. On montrera quels sont les parcours qui, au fil des générations, marquent le pas et ceux qui, a contrario, se diffusent. 
Nos analyses s'appuient sur un modèle de microsimulation. Il a permis, dans chaque génération, de reconstituer (et de prévoir pour les générations futures) les parcours matrimoniaux individuels à partir des probabilités de survenue des divers événements démographiques (mortalité, mariage, divorce, veuvage) qui modèlent les destins de chacun. La microsimulation permet ainsi de reconstruire des biographies individuelles, et par là même l'histoire des générations, sans qu'il soit pour autant nécessaire de collecter les données pour chaque individu de sa naissance à son décès.

Cette approche biographique permet, de plus, de prendre en compte la diversité des cheminements individuels possibles. Par exemple, compte tenu de la mortalité différentielle selon l'état matrimonial (Manzoli et collab., 2007), les durées de vie dans le mariage ont été successivement évaluées pour ceux qui ont passé toute leur vie après 60 ans dans ce statut, mais également pour ceux qui sont devenus veufs ou divorcés après 60 ans. Cette étude se concentre sur les personnes âgées de 60 ans et plus et nées entre 1900 et 1950 . Ce seuil de 60 ans a été choisi car en France les personnes ayant franchi cet âge partagent largement un même statut d'anciens actifs ${ }^{1}$. Ces 50 années retracent l'histoire de la vieillesse en France depuis les années 1960 et donnent un éclairage sur les évolutions attendues durant les prochaines décennies. Compte tenu des générations étudiées, cet article analyse principalement le statut matrimonial légal. Toutefois, la situation de fait est également prise en compte dans les générations les plus récentes (1940 et 1950) dans lesquelles la situation légale des personnes âgées ne reflète plus totalement la réalité.

\section{MÉTHODE ET SOURCES}

Pour reconstituer les histoires matrimoniales des hommes et des femmes nés entre 1900 et 1950, un modèle de microsimulation dynamique et par génération a été élaboré. Les modèles de microsimulation sont utilisés de longue date par les démographes. C'est à Guy Orcutt (Orcutt et collab., 1961) que l'on doit le premier modèle comportant un module démographique détaillé. Depuis, de nombreux autres modèles biologiques et démographiques ont été construits (Hyrenius et Adolfsson, 1964; Leridon, 1977; Sheps et collab., 1973), la démographie historique ayant également utilisé cette méthode pour reconstituer les populations passées (Hammel et

1. L'âge légal à la retraite a été modifié en 2011 et est passé de 60 ans à 62 ans. Toutefois, en 2010, seuls $17 \%$ des 60-64 ans occupaient un emploi. 
collab., 1990 ; Ruggles, 1987; Smith et Oeppen, 1993) et analyser les tendances démographiques sur le long terme. L'un des intérêts majeurs de cette méthode pour les démographes est qu'elle permet d'étudier le réseau familial grâce à la possibilité qu'elle donne d'intégrer tous les liens familiaux possibles (Duée et collab., 2005; Hammel et collab., 1981; Le Bras, 1973; Pennec, 1996; Tomassini et Wolf, 2000 ; Van Imhoff et Post, 1997).

Le modèle utilisé simule les événements démographiques essentiels que les personnes sont susceptibles de rencontrer de l'âge de 60 ans à leur décès avec plus de détails que ne le feraient d'autres méthodes telles que les tables ou les projections multi-états (Palloni, 2005; Van Imhoff et Keilman, 1991; Willekens, 1982). Un exemple type est la prise en compte de la différence d'âge entre conjoints : au lieu d'utiliser une simple moyenne, elle a été intégrée en tenant compte de distributions de différences d'âge selon l'âge à l'union et le type d'union (légal ou de fait; premier mariage ou remariage...). En outre, l'un des avantages de la microsimulation est de pouvoir aisément ajouter des covariables pour affiner les estimations, ou intégrer des modules/événements supplémentaires. Le modèle utilisé est une microsimulation dynamique et longitudinale : dynamique car les individus évoluent au cours de leur cycle de vie (en l'occurrence de leur soixantième année à leur décès) en fonction des événements qu'ils peuvent connaître, longitudinale car on s'intéresse à différentes cohortes que l'on suit sur une partie de leur cycle de vie et non à l'ensemble de la population que l'on simule sur une période de temps. Le modèle retenu est un modèle ouvert, c'est-à-dire avec un suivi des individus sans qu'ils aient d'éventuels liens entre eux. Ainsi, lorsqu'une union est simulée, les caractéristiques du conjoint sont affectées à l'individu étudié et déterminées en fonction de probabilités. En revanche dans un modèle fermé les deux époux font partie de la population et il y a appariement entre eux au sein des personnes non en couple de la population. Cet appariement est effectué par optimisation (Bacon et Pennec, 2008; Bouffard et collab., 2001; Perese, 2002). Dans un modèle dynamique de microsimulation, on détermine la survenue de chacun des événements l'un après l'autre (plus ou moins indépendamment) et l'ordre choisi n'est pas neutre sur les évolutions, en particulier en cas d'événements concurrents. Pour pallier cet inconvénient et pouvoir le considérer comme négligeable, la simulation a été faite par pas mensuel.

La population de départ est un échantillon de $3 \%$ de la population à 60 ans des générations 1895 à 1955, le modèle la faisant « vieillir » de cet âge jusqu'à son extinction (voir annexe méthodologique) en simulant les événements démographiques que les personnes peuvent connaître soit la mortalité (et le veuvage), la nuptialité et la divortialité. Pour la génération 
1900, la simulation se base sur des données observées pour l'ensemble du cycle de vie alors que pour la génération 1950, la simulation repose principalement sur des taux projetés, les individus n'ayant atteint l'âge de 60 ans que la dernière année d'observation (2005). Plus généralement, dans les générations récentes l'estimation des étapes de vie manquantes repose sur des extrapolations des évolutions observées les dix dernières années. En outre, compte tenu de la diffusion des situations conjugales de fait, leurs formations et dissolutions ont été simulées à partir de la génération 1940.

Nos sources principales sont les données d'état civil, soit le nombre annuel de décès, de mariages (remariages), de divorces et de veuvages par sexe, âge et statut matrimonial actuel pour les décès et statut matrimonial précédent pour les mariages. Le niveau de détail des données est variable au cours du temps. Dans un premier temps, ces données ont été harmonisées (séparation des veuvages et divorces pour les années où ces deux événements étaient comptabilisés ensemble) et complétées (passage de groupes d'âge en année d'âge pour certaines périodes en particulier avant 1950) afin d'obtenir des distributions par sexe, année d'âge et situation matrimoniale. Pour chaque événement, les taux par âge ont été calculés puis transformés en taux par génération. Pour définir les tendances du futur, les évolutions passées de la mortalité, nuptialité et divortialité ont été prolongées tendanciellement, ou de manière raisonnée lorsque la tendance ne paraissait pas se poursuivre pour de nombreuses années (baisse de la mortalité pour certains âges et/ou certains états matrimoniaux). Ces hypothèses et les simulations ont été faites par génération mais, par souci de clarté, les résultats sont présentés par groupe de dix générations. Par exemple, les résultats de la génération 1900 correspondent en fait à une moyenne des résultats des générations 1895 à 1904 incluses, pour 1910 les résultats des générations 1905-1914 ayant été agrégés, etc. (pour plus de précisions, voir l'annexe méthodologique).

\section{Principales évolutions passées et futures}

La situation matrimoniale à 60 ans dépend de la nuptialité (mariage et remariage), de la divortialité, du veuvage et de la mortalité par état matrimonial tout au long de la vie. Mariages, remariages et divorces restent rares passé 60 ans, c'est surtout la mortalité par état matrimonial qui va modeler les parcours à ce stade de la vie et décider du statut matrimonial au décès.

Mortalité : Quels que soient l'âge et le sexe, les personnes mariées ont un risque plus faible de décéder que leurs homologues non mariés ${ }^{2}$ : la

2. Toutefois, à partir de 80-85 ans, les différences selon l'état matrimonial sont très faibles. 
surmortalité des personnes non mariées tient aux effets de sélection/ protection du mariage, effets plus importants chez les hommes. Autrement dit, la surmortalité des veufs, des célibataires et des divorcés est beaucoup plus forte chez les hommes que chez les femmes. Par exemple, à 60 ans dans la génération 1945 (la dernière observée), côté masculin, les quotients de mortalité des veufs, célibataires ou divorcés sont plus de deux fois supérieurs à ceux des mariés; côté féminin, ce coefficient est inférieur à 1,5 chez les veuves ou divorcées et, sauf dans les générations récentes, n’atteint pas 2 chez les célibataires (figure 1). De plus, au fil des générations et conformément à d'autres recherches (Murphy et collab., 2007), les écarts de

FIGURE 1 Évolution des rapports entre les quotients de mortalité des veufs, célibataires ou divorcés et ceux des mariés à divers âges selon les générations et le sexe

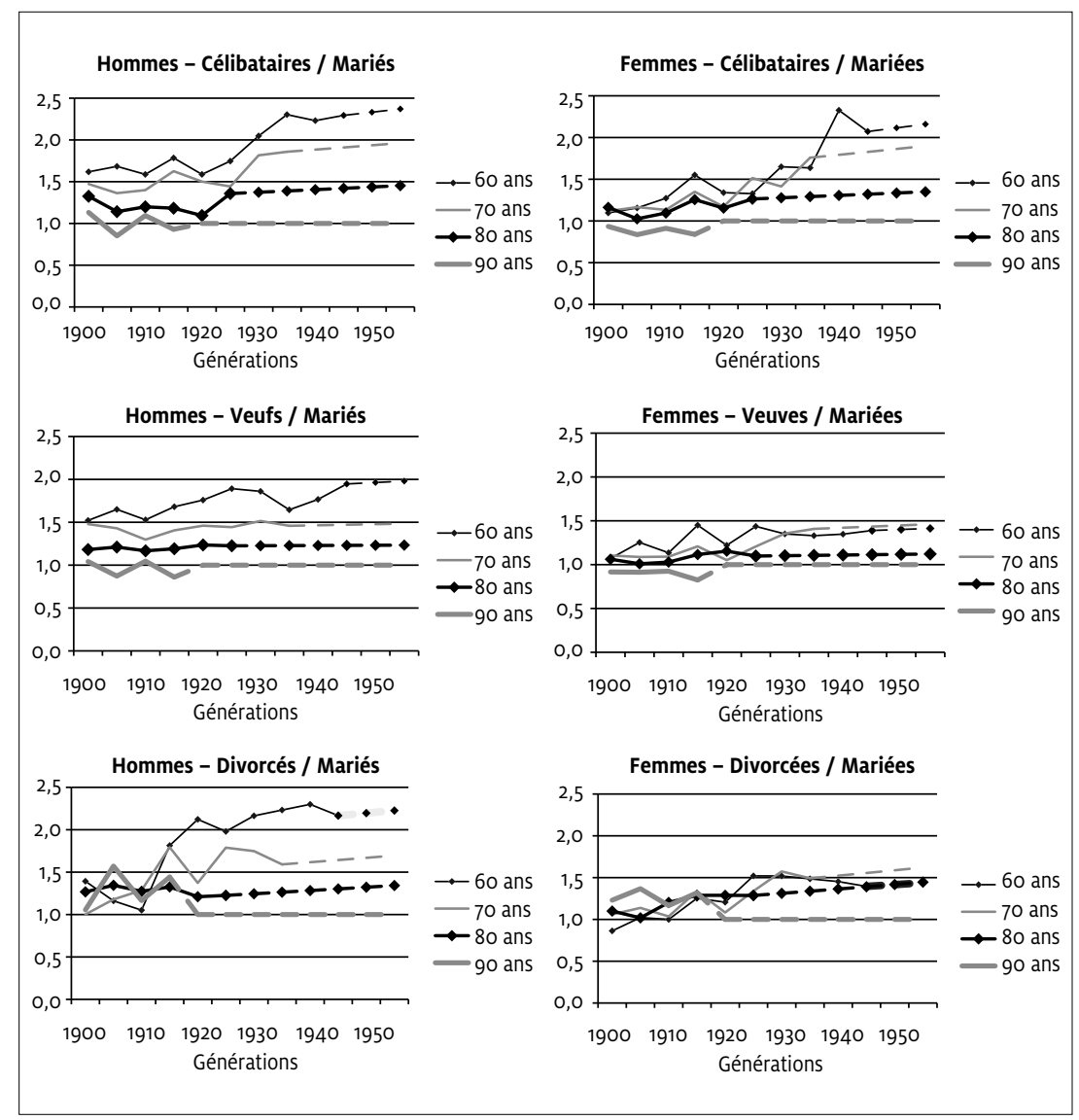

Note: les projections sont représentées par des pointillés. 
mortalité par état matrimonial se sont creusés. Cette tendance est plus accusée chez les hommes que chez les femmes et pour tous aux âges moins élevés, peu de changements apparaissant chez les très âgés.

Nos hypothèses sur les évolutions futures prolongeant les tendances observées les dix dernières années, une hiérarchie claire se dessine dans les évolutions entre les générations 1900 et 1950 (figure 2). Pour les deux sexes et quel que soit l'âge, la mortalité des mariés est celle qui a le plus diminué. Chez les femmes, entre 60 et 80 ans, cette décroissance est suivie de celle des veuves, l'intensité de la baisse de la mortalité des célibataires et divorcés étant du même ordre de grandeur mais derrière celle des deux précédents états matrimoniaux. La hiérarchie est la même chez les hommes, mais seuls les divorcés se distinguent très nettement par des gains beaucoup plus faibles. En outre, quel que soit l'état matrimonial, la baisse de la mortalité a été plus forte chez les femmes que chez les hommes : ainsi, de la génération 1900 à la génération 1950, les quotients de mortalité féminins à 60 ou 65 ans ont diminué de plus de $50 \%$ contre moins de $40 \%$ côté masculin.

FIGURE 2 Évolution des quotients de mortalité entre la génération 1950 et la génération 1900 (quotients 1950/quotients 1900) par âge, état matrimonial et sexe

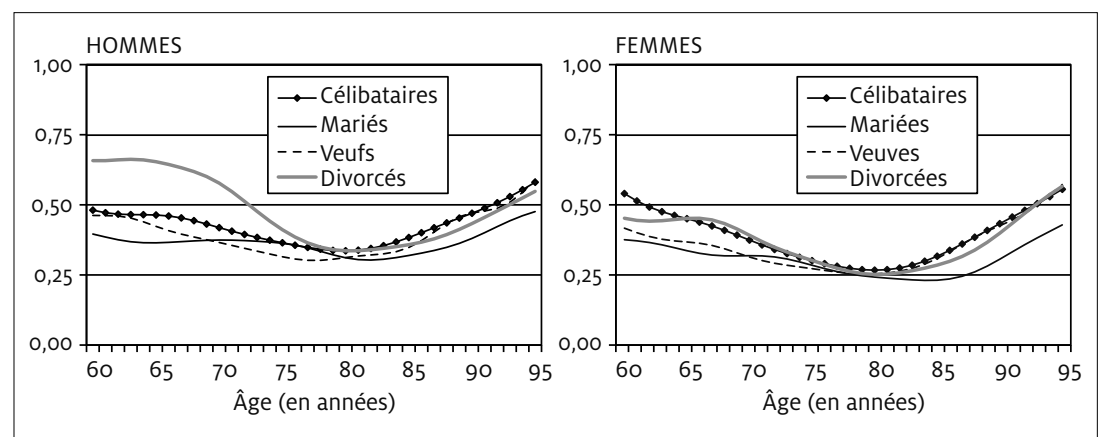

En matière de constitution et de dissolution des couples, au fil des générations, les tendances majeures - et bien connues — sont la diminution des mariages, la croissance des divorces et le recul du veuvage.

Nuptialité : La probabilité de se marier après 50 ans est très faible et, de plus, elle diminue régulièrement avec l'âge : quelle que soit la génération, passé 60 ans, moins de $0,5 \%$ des célibataires se sont mariés dans l'année. Se remarier est un événement qui devient, également, de plus en plus rare avec l'avancée en âge. Les divorcés se remarient plus facilement que les veufs, mais la probabilité de refaire sa vie est toujours moindre pour les 
femmes, particulièrement en cas de veuvage. Ainsi, on a pu montrer (Delbès et Gaymu, 2003b) que dans la génération 1930, moins de 0,2 \% des veuves se sont remariées dans leur $60^{\mathrm{e}}$ année contre $1,1 \%$ des veufs $(0,9 \%$ et $2,7 \%$ chez les divorcés).

Divortialité : Non seulement les personnes âgées d'aujourd'hui ont plus souvent divorcé avant 60 ans que leurs aînées mais, passé cet âge, elles tendent davantage à rompre leur union : ainsi, de la génération 1900 à la génération 1940, la probabilité des hommes de divorcer à 60 ans est passée de 1 à 4 pour mille. Pour les hommes comme pour les femmes, cette probabilité diminue avec l'avancée en âge : dans la génération 1930, elle est à peine supérieure à 1 pour mille à 70 ans (tableau 1 ).

TABLEAU 1 Évolution des taux de divorce par âge, sexe et génération (pour 10 ooo)

\begin{tabular}{|l|c|c|c|c|c|c|}
\hline \multirow{2}{*}{ Générations } & \multicolumn{3}{|c|}{ Hommes } & \multicolumn{3}{c|}{ Femmes } \\
\cline { 2 - 7 } & $\mathbf{6 0}$ ans & $\mathbf{7 0}$ ans & $\mathbf{8 0}$ ans & $\mathbf{6 0}$ ans & $\mathbf{7 0}$ ans & $\mathbf{8 0}$ ans \\
\hline $\mathbf{1 9 0 0}$ & 8,7 & 4,6 & 3,1 & 9,3 & 4,8 & 2,8 \\
\hline $\mathbf{1 9 1 0}$ & 12,0 & 5,2 & 3,7 & 13,0 & 5,4 & 3,2 \\
\hline $\mathbf{1 9 2 0}$ & 22,0 & 11,0 & 6,6 & 23,0 & 12,0 & 5,9 \\
\hline $\mathbf{1 9 3 0}$ & 23,0 & 11,0 & 5,9 & 25,0 & 11,0 & 5,7 \\
\hline $\mathbf{1 9 4 0}$ & 38,0 & 21,0 & 6,2 & 41,0 & 22,0 & 6,4 \\
\hline $\mathbf{1 9 5 0}$ & 75,0 & 25,0 & 6,2 & 76,0 & 28,0 & 6,4 \\
\hline
\end{tabular}

Note: les projections sont indiquées en italique.

Veuvage: Conséquence, pour l'essentiel, de la surmortalité masculine, le risque de veuvage est beaucoup plus fort pour les femmes. Presque nul avant 50 ans, il augmente modérément jusqu'à 60 ans pour connaître ensuite une forte accélération reproduisant les courbes de mortalité : dans la génération 1930, 2,3\% des femmes sont devenues veuves $(0,5 \%$ des hommes, veufs) dans leur $60^{\mathrm{e}}$ année, cette proportion atteignant $8,1 \%$ dans leur $80^{\mathrm{e}}$ (2,8 \% pour les hommes). La probabilité de voir disparaitre son conjoint diminue régulièrement depuis plusieurs décennies : ainsi à 60 ans, le risque des femmes a été divisé par plus de 2 entre les générations 1900 et 1940 (soit respectivement $3,5 \%$ et $1,7 \%$ ).

Compte tenu de nos hypothèses de prolongement des tendances passées en matière de mortalité, cette évolution est appelée à se poursuivre à l'avenir : par exemple, le risque de devenir veuve à 70 ans des femmes nées en 1940 pourrait être inférieur à celui observé à 60 ans chez celles nées 30 ans plus tôt. La baisse de la mortalité ayant été plus forte chez les femmes 
mariées que chez leurs homologues masculins, l'âge moyen d'entrée dans le veuvage a davantage reculé chez les hommes que chez les femmes : égal à 73,0 ans pour les premiers et 70,5 ans pour les secondes dans la génération 1900, il pourrait atteindre respectivement 81,7 et 77,6 ans dans la génération 1950.

TABLEAU 2 Évolution du risque de veuvage selon l'âge, le sexe et la génération (pour 100)

\begin{tabular}{|l|c|c|c|c|c|c|c|c|}
\hline \multirow{2}{*}{ Générations } & \multicolumn{4}{|c|}{ Hommes } & \multicolumn{4}{c|}{ Femmes } \\
\cline { 2 - 10 } & $\mathbf{6 0}$ ans & $\mathbf{7 0}$ ans & $\mathbf{8 0}$ ans & $\mathbf{9 0}$ ans & 60 ans & 70 ans & $\mathbf{8 0}$ ans & 90 ans \\
\hline $\mathbf{1 9 0 0}$ & 1,1 & 2,4 & 5,6 & 10,8 & 3,5 & 6,9 & 13,9 & 22,3 \\
\hline $\mathbf{1 9 1 0}$ & 0,8 & 1,7 & 4,3 & 11,3 & 3,3 & 6,1 & 11,0 & 19,3 \\
\hline $\mathbf{1 9 2 0}$ & 0,7 & 1,3 & 3,5 & 8,4 & 2,8 & 5,0 & 10,5 & 16,5 \\
\hline $\mathbf{1 9 3 0}$ & 0,5 & 1,1 & 2,8 & 7,6 & 2,3 & 4,1 & 8,1 & 15,8 \\
\hline $\mathbf{1 9 4 0}$ & 0,4 & 0,7 & 2,0 & 7,3 & 1,7 & 3,2 & 7,1 & 14,6 \\
\hline $\mathbf{1 9 5 0}$ & 0,3 & 0,6 & 1,6 & 6,5 & 1,4 & 2,6 & 6,3 & 14,3 \\
\hline
\end{tabular}

Note: les projections sont indiquées en italique.

\section{LA SITUATION MATRIMONIALE À 60 ANS ET CELLE AU DÉCÈS}

Jusqu'à la génération 1930, environ $83 \%$ des hommes étaient mariés à 60 ans (figure 3). Ensuite, cette proportion s'est mise à décliner et elle pourrait n'être que de $74 \%$ dans la génération 1950 en raison de la croissance de la part des divorcés (3\% dans la génération 1930 contre $13 \%$ dans la génération 1950). Chez les femmes, l'évolution au fil des générations est un peu différente : celles des générations 1900 à 1930 ont atteint l'âge de 60 ans plus souvent mariées (respectivement $62 \%$ et $70 \%$ ) et moins souvent veuves ( $24 \%$ et $17 \%$ ). Dans les générations suivantes, la part des mariées pourrait rester quasiment inchangée ( $68 \%$ dans la génération 1950), le déclin de celle des veuves (7\% dans la génération 1950) et la croissance de celle des divorcées ( $5 \%$ dans la génération 1930 contre $16 \%$ dans la génération 1950) se compensant.

Conformément à de nombreuses autres recherches, ces résultats confirment qu'à 60 ans, les distributions par état matrimonial des hommes et des femmes se sont rapprochées au cours du temps : dans la génération 1900 , les hommes de 60 ans étaient 1,3 fois plus souvent mariés que les femmes, ce rapport ayant chuté à 1,1 à partir de la génération 1940. 
La situation matrimoniale à 60 ans n'est évidemment pas immuable; les personnes mariées à cet âge ne le resteront pas nécessairement jusqu'à la fin de leurs jours. Sur ce point, hommes et femmes ont des destins très différents. Si à 60 ans tous sont majoritairement mariés, leurs situations conjugales deviennent radicalement différentes au décès (figure 3 ) : la majorité des hommes resteront mariés jusqu'à leur décès (entre $52 \%$ et $59 \%$ selon les générations) alors que la majorité des femmes ( $57 \%$ à $71 \%)$ mourront veuves. Il n'y a pas d'évolution marquante jusqu'à la génération 1930, mais ensuite les hommes et les femmes meurent moins souvent veufs ou mariés et plus souvent divorcés et, dans une moindre mesure, célibataires.

FIGURE 3 Distribution des situations matrimoniales (\%) à 60 ans et au décès selon le sexe et la génération

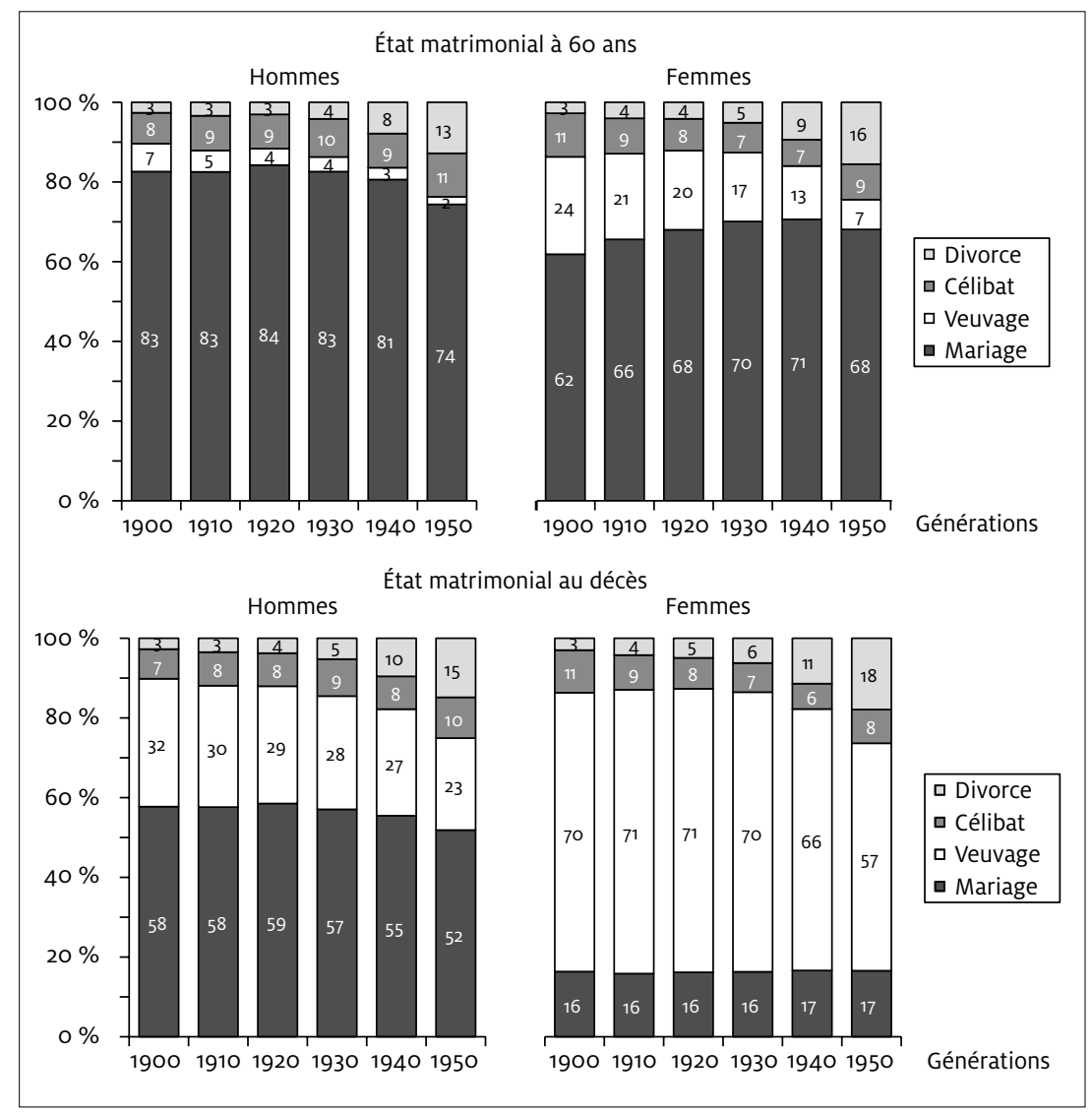


Hommes et femmes se rejoignent sur un autre point : ceux devenus veufs vivront le plus longtemps, et ceux restés mariés jusqu'à la mort le moins longtemps (figure 4). Chez les femmes l'écart est, selon les générations de 10 à 11 ans et chez les hommes de l'ordre de 7 à 8 ans : à chaque âge et quelle que soit la génération, la survie des mariés est la plus forte mais décéder marié signifie décéder précocement. Par ailleurs, la surmortalité masculine conduit généralement les femmes à survivre à leur conjoint : celles qui ne connaissent pas ce parcours sont donc sélectionnées négativement; les hommes survivant à leur épouse l'étant, à l'inverse, positivement. En conséquence, alors qu'en moyenne l'espérance de vie à 60 ans des femmes est supérieure d'environ 6 ans à celle des hommes, cet écart n'est que de 2 ans chez ceux qui mourront veufs. Quant aux femmes restées mariées jusqu'à leur décès, elles vivront moins longtemps que les hommes dans cette situation (figure 4).

Les espérances de vie des divorcés et célibataires sont proches mais très inférieures à la moyenne côté masculin et à peine plus faibles côté féminin. Les écarts d'espérance de vie selon le sexe sont donc beaucoup plus accusés que pour les mariés et veufs (plus de 7 ans dans quasiment toutes les générations (figure 4). Ils sont, en outre, du même ordre de grandeur que l'on se situe à 60 ans ou au décès : dans ces générations et à ces âges, quasiment toutes les personnes célibataires ou divorcés à 60 ans le resteront jusqu'à leur décès.

FIGURE 4 Évolution de la durée de vie (en années) à 60 ans en fonction de l'état matrimonial à 60 ans et au décès selon la génération et le sexe

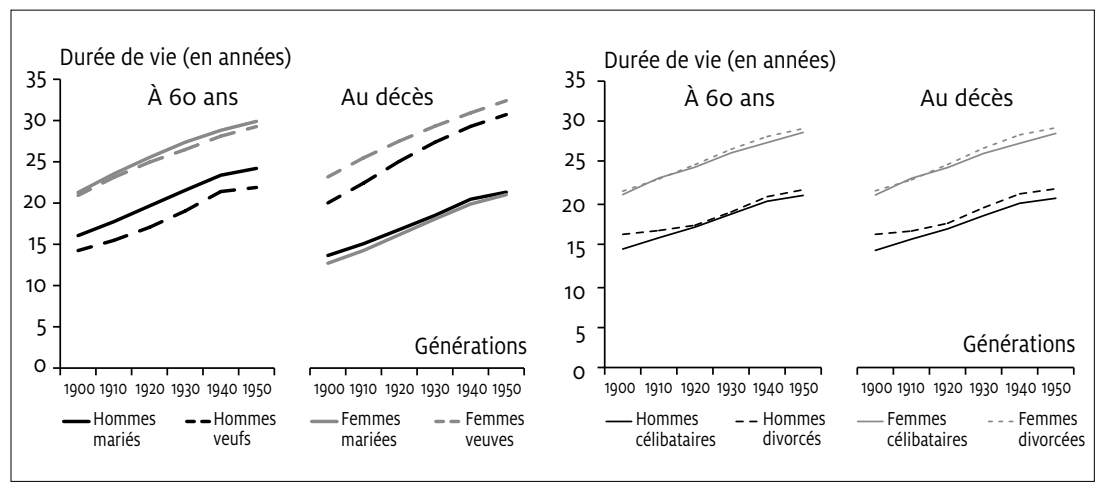

Après ce survol des différences dans le statut des hommes et des femmes à 60 ans et au moment du décès, nous allons analyser les divers parcours susceptibles d'être suivis entre ces deux étapes. La microsimulation est un outil précieux en ce domaine puisqu'elle permet de reconsti- 
tuer les biographies individuelles grâce à la prise en compte de tous les événements qui peuvent ponctuer la vie après 60 ans.

\section{L'ÉVOLUTION DE LA DURÉE DE VIE SELON LES PARCOURS MATRIMONIAUX}

Nous allons tout d'abord analyser l'évolution de la situation de ceux qui, de 60 ans à leur mort, sont restés dans la même situation conjugale (tableaux 3 et 4 ; figure 5).

\section{Être marié de 60 ans au décès}

Ce parcours pourrait encore concerner la majorité des hommes dans l'avenir même si sa fréquence diminue $(49,2 \%$ dans la génération 1950 contre 56,3 \% dans celle de 1900, tableau 3). En revanche, il ne touche qu'une très faible fraction des femmes, quelle que soit la génération (environ 15,0 \%). Entre les générations nées en 1900 et 1950, la mortalité des mariés est celle qui devrait le plus diminuer, conduisant les hommes et les femmes restés mariés jusqu'à leur décès à connaître la plus forte augmentation, en termes relatifs, de leur durée de vie. La progression, liée à la probabilité de chacun des conjoints de survivre, devrait être identique pour les hommes et les femmes (7,5 ans, tableau 4). Les hommes continueront donc à avoir une durée de vie supérieure d'un an à celle des femmes (21,1 contre 19,9 ans dans la génération 1950) notamment car ils ont une plus forte probabilité d'avoir une épouse de moins de 60 ans.

\section{Être veuf de 60 ans au décès}

Être veuf de 60 ans jusqu'au décès est un parcours en nette perte de vitesse surtout pour les femmes : 24,3\% d'entre elles étaient dans ce cas dans la génération 1900 contre $17,2 \%$ dans la génération 1930 et 7,4 \% dans la génération 1950 (respectivement $6,3 \%, 3,4 \%$ et $1,7 \%$ pour les hommes). Dans cette configuration matrimoniale, la durée de vie des femmes se situe dans la moyenne, mais en deçà de la moyenne pour les hommes, le veuvage étant, pour eux, associé avec une plus forte surmortalité. En outre, durant la période considérée, les évolutions devraient être proches de la moyenne, conduisant à un léger creusement des écarts dans les durées de veuvage des hommes et des femmes : 7,1 ans dans la génération 1900 contre 7,8 ans dans la génération 1950 . 
TABLEAU 3 Distribution de la population (\%) en fonction de l'état matrimonial à 60 ans et au décès selon le sexe et la génération

\begin{tabular}{|c|c|c|c|c|c|c|c|c|c|c|c|c|c|}
\hline \multicolumn{2}{|c|}{ État matrimonial } & \multicolumn{6}{|c|}{ Hommes } & \multicolumn{6}{|c|}{ Femmes } \\
\hline À 60 ans & Au décès & 1900 & 1910 & 1920 & 1930 & 1940 & 1950 & 1900 & 1910 & 1920 & 1930 & 1940 & 1950 \\
\hline Célibataire & Célibataire & 7,4 & 8,4 & 8,3 & 9,3 & 8,2 & 10,2 & 10,7 & 8,7 & 7,7 & 7,3 & 6,4 & 8,5 \\
\hline \multirow[t]{3}{*}{ Marié } & Marié & 56,3 & 56,2 & 57,4 & 56,0 & 53,8 & 49,2 & 15,9 & 15,3 & 15,7 & 15,9 & 15,8 & 14,8 \\
\hline & Veuf & 25,7 & 25,6 & 25,6 & 25,0 & 24,0 & 21,3 & 45,6 & 49,8 & 51,3 & 52,8 & 52,1 & 49,4 \\
\hline & Divorcé & 0,5 & 0,7 & 1,2 & 1,7 & 2,8 & 3,9 & 0,4 & 0,5 & 1,0 & 1,4 & 2,7 & 3,9 \\
\hline Veuf & Veuf & 6,3 & 4,8 & 3,8 & 3,4 & 2,7 & 1,7 & 24,3 & 21,4 & 19,8 & 17,2 & 13,3 & 7,4 \\
\hline Divorcé & Divorcé & 2,2 & 2,8 & 2,5 & 3,6 & 6,7 & 10,8 & 2,5 & 3,7 & 3,9 & 4,8 & 8,7 & 13,9 \\
\hline Autres & & 1,5 & 1,5 & 1,2 & 1,2 & 1,8 & 2,9 & 0,6 & 0,6 & 0,6 & 0,6 & 1,0 & 2,1 \\
\hline Ensemble & & 100,0 & 100,0 & 100,0 & 100,0 & 100,0 & 100,0 & 100,0 & 100,0 & 100,0 & 100,0 & 100,0 & 100,0 \\
\hline
\end{tabular}

TABLEAU 4 Durée de vie à 60 ans (en années) selon le parcours conjugal entre 60 ans et le décès en fonction du sexe pour les générations 1900 et 1950

\begin{tabular}{|c|c|c|c|c|c|c|c|c|c|}
\hline \multicolumn{2}{|c|}{ État matrimonial } & \multicolumn{3}{|c|}{ Hommes } & \multicolumn{3}{|c|}{ Femmes } & \multicolumn{2}{|c|}{ Femmes-Hommes } \\
\hline À 60 ans & Au décès & 1900 & 1950 & 1950-1900 & 1900 & 1950 & 1950-1900 & 1900 & 1950 \\
\hline Célibataire & Célibataire & 13,8 & 20,3 & 6,5 & 20,7 & 28,5 & 7,7 & 6,9 & 8,2 \\
\hline \multirow[t]{3}{*}{ Marié } & Marié & 13,5 & 21,1 & 7,6 & 12,5 & 19,9 & 7,4 & $-1,0$ & $-1,2$ \\
\hline & Veuf & 21,5 & 31,4 & 9,9 & 24,3 & 32,9 & 8,6 & 2,8 & 1,5 \\
\hline & Divorcé & 17,5 & 24,1 & 6,6 & 21,9 & 30,3 & 8,4 & 4,4 & 6,2 \\
\hline Veuf & Veuf & 13,8 & 21,5 & 7,7 & 20,9 & 29,3 & 8,4 & 7,1 & 7,8 \\
\hline Divorcé & Divorcé & 15,4 & 20,6 & 5,2 & 21,2 & 28,8 & 7,6 & 5,8 & 8,2 \\
\hline Ensemble & & 15,8 & 23,4 & 7,6 & 21,1 & 29,6 & 8,5 & 5,3 & 6,2 \\
\hline
\end{tabular}


FIGURE 5 Durée de vie à 60 ans des personnes restées dans le même état matrimonial de 60 ans au décès selon l'état matrimonial, le sexe et la génération

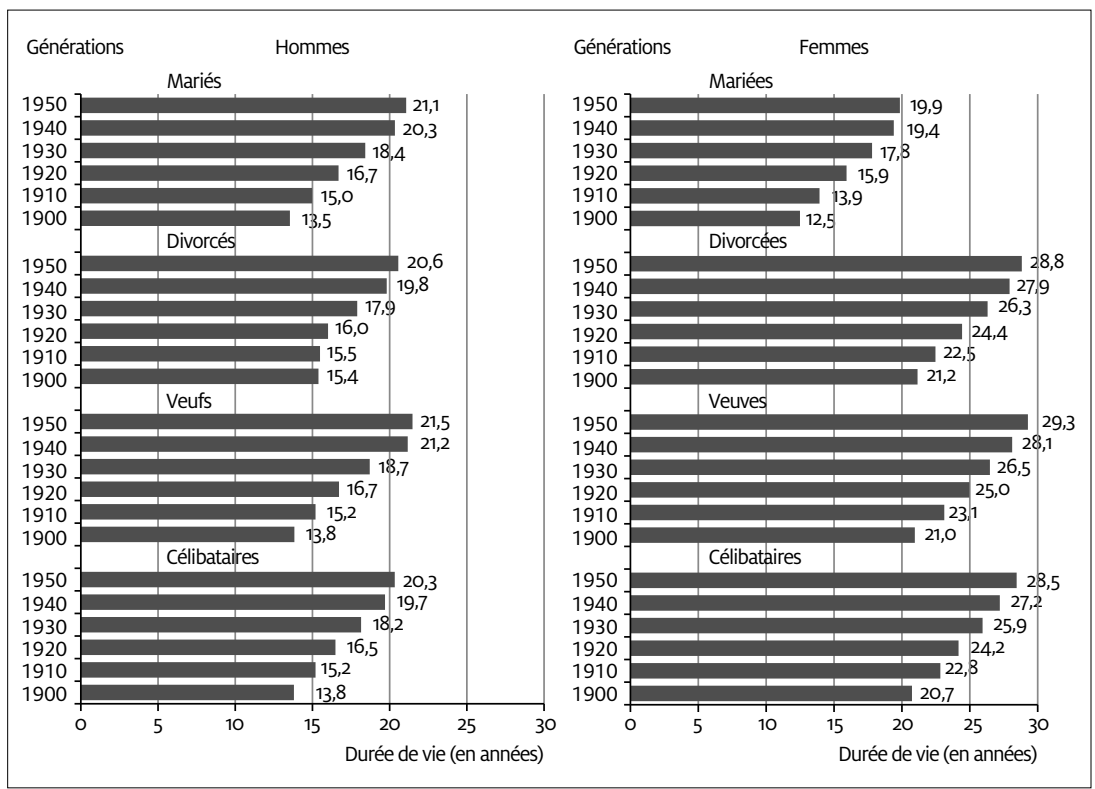

\section{Être divorcé de 60 ans au décès}

C'est la situation matrimoniale susceptible d'augmenter, en valeur absolue, le plus fortement dans l'avenir (10,8 \% des hommes et $13,9 \%$ des femmes de la génération 1950 contre respectivement $2,2 \%$ et $2,5 \%$ dans la génération 1900, tableau 3). Au fil des générations, la survie des hommes divorcés est celle qui a le moins augmenté : ceux qui sont restés divorcés, autrement dit ceux qui ne se sont pas remis en couple, seraient donc sélectionnés de plus en plus négativement ${ }^{3}$. En conséquence, les écarts de durée de vie à 60 ans selon le sexe devraient se creuser beaucoup plus que dans les autres parcours matrimoniaux : égal à 5,8 ans dans la génération 1900, l'écart pourrait atteindre 8,2 ans dans la génération 1950. C'est dans cette configuration matrimoniale que les écarts de durée de vie entre hommes et femmes sont susceptibles de s'accroître le plus fortement.

3. La figure 5 montre clairement que ceci est surtout vrai pour les générations anciennes, la mortalité des hommes et des femmes divorcés ayant évolué de façon plus comparable plus récemment. 


\section{Être célibataire de 60 ans au décès}

Ce parcours matrimonial va se diffuser à l'avenir chez les hommes en particulier ${ }^{4}$ : dans la génération 1950, 10,2 \% d'entre eux $(8,5 \%$ des femmes) pourraient être dans ce cas. La durée de vie des célibataires, surtout masculins, est inférieure à la moyenne et elle fait partie de celles qui devraient le moins augmenter: de 6,5 ans chez les hommes et 7,7 ans chez les femmes entre les générations 1900 et 1950. De plus, la sélection beaucoup plus négative des hommes que des femmes a tendance à s'accentuer au fil des générations : l'écart entre leurs durées de vie, déjà parmi les plus forts dans la génération 1900 (6,9 ans), pourrait se creuser encore (8,1 ans dans la génération 1950).

\section{Être marié à 60 ans et décéder veuf}

Quelle que soit la génération, c'est le destin le plus fréquent des femmes (entre $45,6 \%$ et 52,8 \% d'entre elles), alors qu'environ $25 \%$ seulement des hommes sont dans ce cas. Chez eux, cette proportion, restée longtemps stable, a légèrement baissé à partir de la génération 1930. En revanche, chez les femmes, elle a progressé jusqu'aux générations 1930-1940 à la suite du recul du veuvage avant 60 ans 5 . Dans les générations plus récentes, hommes et femmes mariés devraient décéder relativement moins souvent veufs car une fraction plus élevée mourront divorcés (tableau 3).

Quelle que soit la génération, c'est dans ce cas de figure que les durées de vie des hommes et des femmes sont les plus longues - 31,4 ans pour les hommes et 32,9 ans pour les femmes dans la génération 1950 - et parmi les plus proches (tableau 4). Toutefois, dans ce parcours, les hommes passent l'essentiel de leur vie en couple (entre $60 \%$ et $70 \%$ selon les générations) alors que les femmes vivent quasiment autant d'années dans le veuvage que dans le mariage, conséquence de leur âge plus précoce au veuvage. Pour les deux, l'essentiel des gains provient des années dans le mariage (quel que soit le rang du mariage) (figure 6). Ces gains ont été plus forts pour les hommes $(8,5$ années contre 7,1 ans des générations 1900 à 1950), en raison du plus fort recul de leur âge d'entrée dans le veuvage. Au fil des générations, la part des années passées dans le veuvage n'a cessé de diminuer pour les deux sexes et elle pourrait devenir minoritaire chez

\footnotetext{
4. Les générations féminines nées autour de 1900 ayant subi un fort célibat en raison de la guerre.

5. La tendance est identique chez les hommes mais beaucoup moins accusée car ils sont peu touchés par le veuvage.
} 
les femmes à partir de la génération 1940. La durée passée dans le veuvage n'a augmenté que pour les générations nées avant 1920 (d'une année et demie environ), pour les suivantes elle devrait rester remarquablement stable (de l'ordre de 9 ans pour les hommes et 15 pour les femmes).

Conséquence, les différences entre situations masculines et féminines se sont creusées : dans la génération 1900, les hommes passaient 2,9 ans de plus dans le mariage que les femmes contre 4,2 ans dans la génération 1950. Dans le même temps, le surplus d'années de vie dans le veuvage des femmes devrait rester identique (5,7 ans).

FIGURE 6 Durée de vie à 60 ans des mariés à 60 ans ayant connu le veuvage entre 60 ans et leur décès selon le sexe et la génération

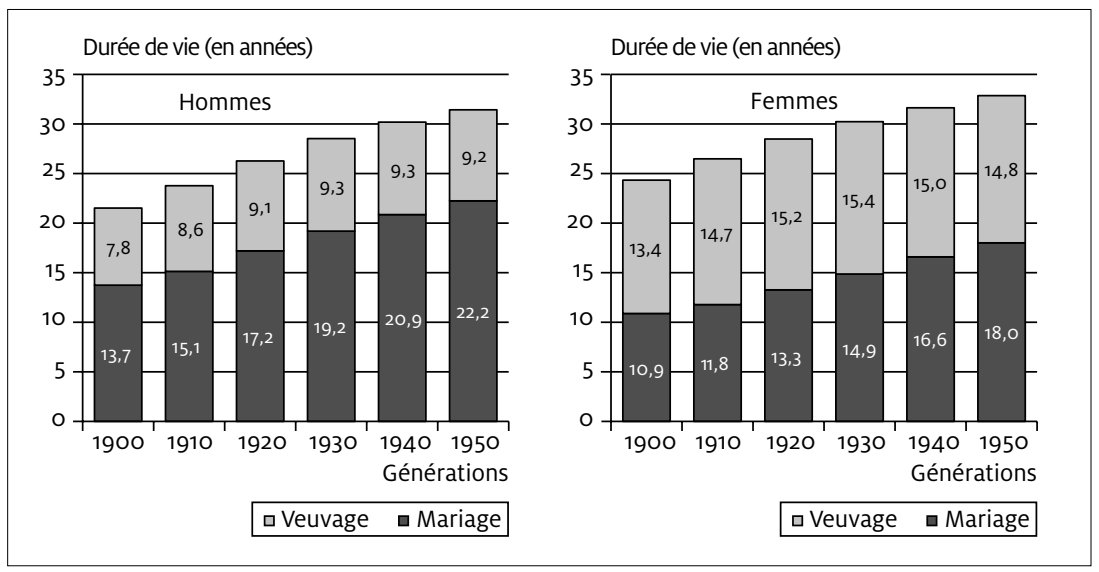

\section{Mourir divorcé, en ayant divorcé après 60 ans}

Malgré sa progression, ce parcours matrimonial ne devrait concerner que peu d'hommes et de femmes dans l'avenir (3,9\% dans la génération 1950). Si dans ce cas de figure, les hommes passent proportionnellement plus de temps dans le mariage que les femmes (soit environ $35 \%$ contre $25 \%$ quelle que soit la génération), pour les deux l'essentiel des années de vie se déroule dans le divorce (figure 7). De plus, la quasi-totalité des gains au fil des générations sont des années passées dans le divorce, le temps passé dans le mariage étant resté quasiment stable. Il est vrai qu'à ce stade de la vie, l'âge au divorce varie peu et se situe généralement dans la soixantaine.

Au fil des générations, on retrouve ici les mêmes tendances que celles observées précédemment pour les personnes ayant divorcé avant 60 ans. Dans ce parcours conjugal, l'écart d'espérance de vie entre hommes et 
femmes, égal à 4,4 ans dans la génération 1900 pourrait atteindre 6,2 ans dans la génération 1950, soit une des plus fortes augmentations.

FIGURE 7 Durée de vie à 60 ans des mariés à 60 ans ayant connu le divorce entre 60 ans et leur décès selon le sexe et la génération

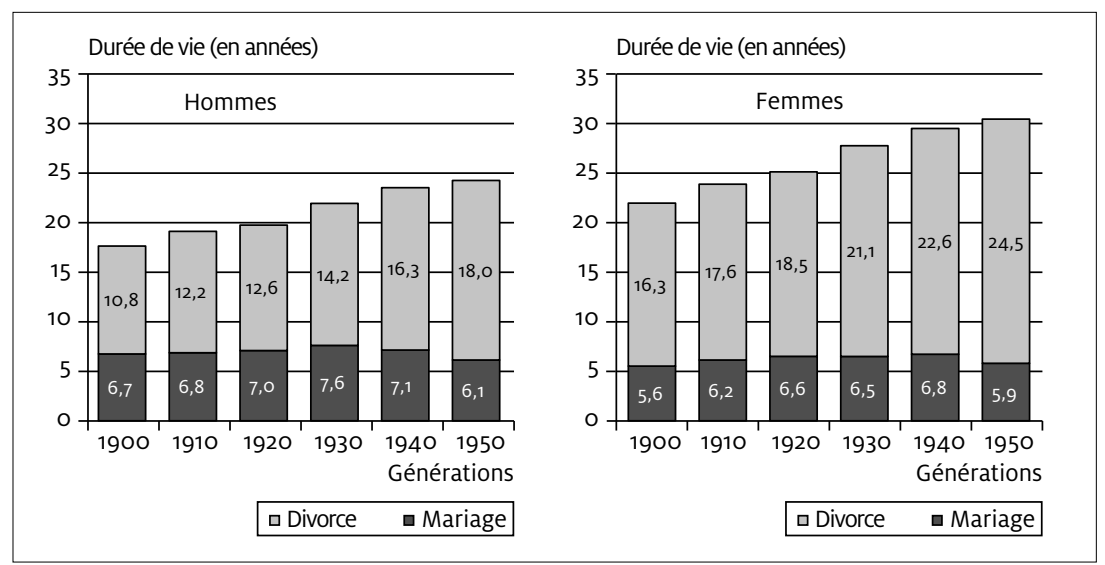

\section{FINALEMENT, QUELLE ÉVOLUTION DE L'ISOLEMENT MATRIMONIAL PASSÉ 60 ANS?}

Quelle que soit la génération, les années de vie des hommes après 60 ans se déroulent essentiellement dans le mariage (de l'ordre de $70 \%$ dans quasiment toutes les générations, figure 8 ). Non seulement leur probabilité de mourir marié est plus élevée que celle des femmes mais même lorsqu'ils meurent veufs, leur durée de vie en couple est plus longue. En revanche, les femmes de toutes les générations ne passent qu'environ $40 \%$ de leur vie après 60 ans dans le mariage, l'essentiel jusque dans les cohortes 1930 étant constitué d'années de veuvage. Pour les 20 générations suivantes, la durée du veuvage devrait diminuer au point de devenir inférieure à celle du mariage dans la génération 1950 (soit respectivement $32 \%$ et $42 \%$ ). L'autre tendance marquante pour les deux sexes est la forte progression de la proportion d'années passées dans le divorce : moins de $6 \%$ jusqu'à la génération 1930 contre $13 \%$ pour les hommes et $17 \%$ pour les femmes de la génération 1950 .

La génération 1930, c'est-à-dire les personnes très âgées d'aujourd'hui, marque une cassure dans les évolutions (tableau 5 et figure 9). Pour les 30 premières générations, les gains de durée de vie à 60 ans se sont faits pour 
l'essentiel dans le mariage et de façon quasi identique pour les hommes et les femmes (respectivement 4 ans et 3,8 ans, tableau 5).

FIGURE 8 Évolution de la part (\%) des années vécues dans les divers états matrimoniaux des personnes de 60 ans selon la génération et le sexe, quel que soit l'état matrimonial à 60 ans et au décès

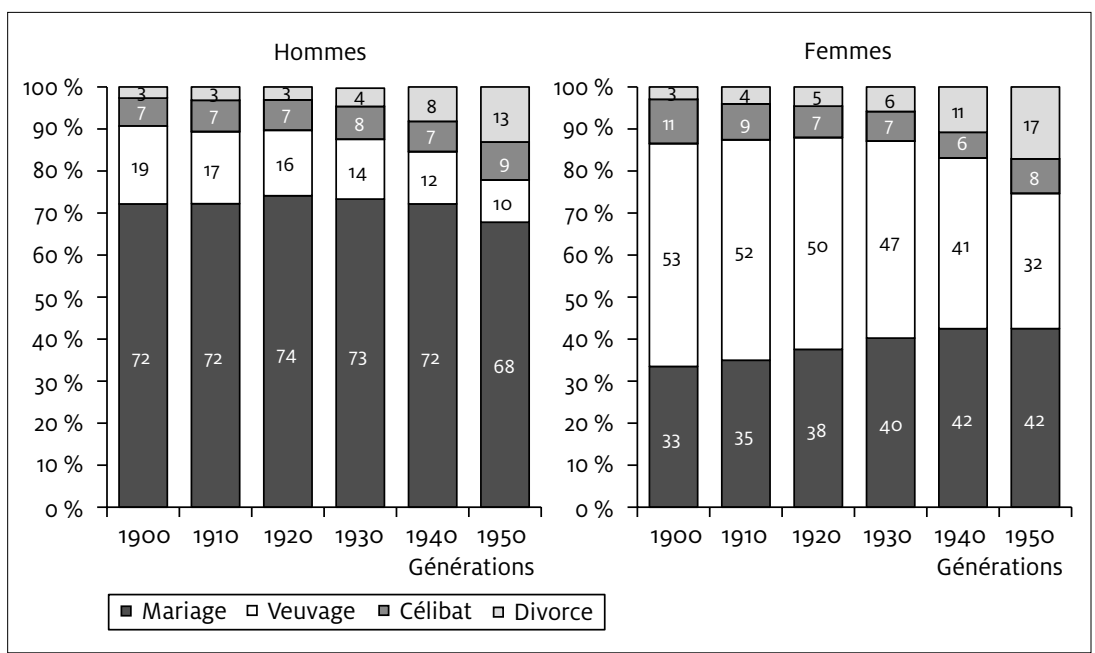

TABLEAU 5 Nombre d'années gagnées dans les divers états matrimoniaux selon le sexe et la génération, quel que soit l'état matrimonial initial et final

\begin{tabular}{|l|c|c|c|c|c|}
\hline Générations & Célibat & Mariage & Veuvage & Divorce & Ensemble \\
\hline 1900-1930 \\
\hline Hommes & 0,7 & 4,0 & 0,1 & 0,5 & 5,3 \\
\hline Femmes & $-0,3$ & 3,8 & 1,5 & 1,0 & 5,9 \\
\hline 1930-1950 \\
\hline Hommes & 0,4 & 0,5 & $-0,6$ & 2,2 & 2,4 \\
\hline Femmes & 0,5 & 1,7 & $-3,2$ & 3,5 & 2,5 \\
\hline 1900-1950 & & & & \\
\hline Hommes & 1,1 & 4,5 & $-0,6$ & 2,6 & 7,7 \\
\hline Femmes & 0,2 & 5,5 & $-1,7$ & 4,4 & 8,5 \\
\hline
\end{tabular}

Par ailleurs, jusqu'à la génération 1930, la durée de vie dans le veuvage est restée stable chez les hommes, alors qu'elle a augmenté de 1,5 an chez les femmes. La durée de vie des veufs, hommes comme femmes, s'est accrue mais cette évolution a été compensée chez les premiers par une 
FIGURE 9 Évolution du nombre d'années vécues dans les divers états matrimoniaux selon la génération et le sexe, quel que soit l'état matrimonial initial et final

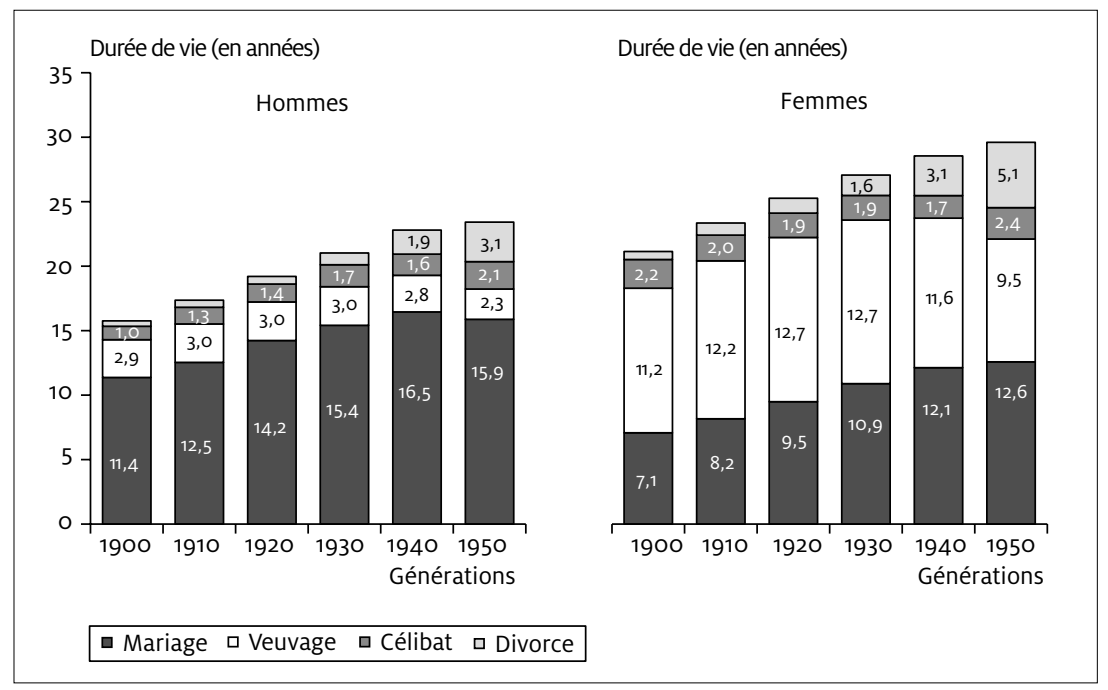

diminution du risque d'être veuf (avant comme après 60 ans) alors que chez les secondes elle s'est doublée d'une augmentation de la fréquence du veuvage après 60 ans.

Enfin, l'augmentation de la durée de vie à 60 ans en tant que divorcé a été plus forte pour les femmes que pour les hommes ( 1 année contre 0,5 an), l'inverse valant pour le célibat $(-0,3$ contre 0,7$)$. Le risque de connaître un divorce a progressé de façon identique mais la durée de vie a davantage augmenté chez les femmes que chez les hommes divorcés. Ce dernier constat vaut aussi chez les célibataires mais une proportion croissante d'hommes est dans cette situation matrimoniale alors que c'est l'inverse pour les femmes.

Au total entre ces générations (1900-1930), le surplus d'espérance de vie des hommes comme des femmes est passé pour l'essentiel dans le mariage mais la durée de vie en isolement matrimonial a plus augmenté chez les femmes (2,2 ans contre 1,2 an).

Cette tendance à vivre plus longtemps mariés devrait se poursuivre après la génération 1930. Compte tenu de l'inégal nombre de générations (20 pour le futur contre 40 pour le passé), les gains sont quasiment identiques pour les femmes (soit 1,7 an contre 3,8 ans), les progrès enregistrés par les hommes connaissant, en revanche, un net ralentissement 
( 0,5 an contre 4 ans). En fait, pour les 20 générations suivantes, le surplus d'espérance de vie sera, pour l'essentiel, composé d'années vécues dans le divorce et plus particulièrement pour les femmes (3,5 et 2,1 années respectivement). Que le divorce ait eu lieu avant ou après 60 ans, la durée de vie des divorcés devrait continuer à s'accroître (en moyenne, de près de 3 ans chez les femmes comme chez les hommes ${ }^{6}$ ), mais mourir divorcé progressera davantage chez les femmes.

Enfin, il faut s'attendre pour les générations futures à une beaucoup plus importante diminution de la durée de veuvage chez les femmes que chez les hommes (soit respectivement 3,2 ans contre 0,6 an). Il est vrai que dans ces générations la fréquence du veuvage a beaucoup plus fortement reculé chez elles.

Au total dans ces générations (1940-1950), l'espérance de vie des hommes et des femmes devrait augmenter de façon quasi identique. Chez les femmes, la baisse de la durée du veuvage et la croissance de celle du divorce se compensent quasiment et l'essentiel de la progression (soit 1,7 sur 2,5 ans) devrait se dérouler dans le mariage. C'est l'inverse chez les hommes, la majeure partie de l'allongement de leur durée de vie (1,9 sur 2,4 ans) devrait être des années d'isolement matrimonial, passées principalement dans le divorce.

Toutefois, pour les personnes âgées comme pour les plus jeunes, la situation matrimoniale légale est de moins en moins représentative de la situation conjugale, autrement dit ne pas être marié signifie de moins en moins souvent ne pas vivre en couple. La cohabitation est essentiellement développée chez les moins de 70 ans et dans les générations les plus récentes. Elle est beaucoup moins répandue chez les femmes (Cassan et collab., 2001) et les divorcés ont les plus fortes chances de se remettre en union (les veufs et les célibataires ayant des probabilités environ deux fois moins élevées). L'introduction de ces données dans le modèle de microsimulation modifie les résultats à double titre (tableau 6).

En termes de proportions, intégrer l'ensemble des unions joue plus en faveur des hommes, ces derniers reformant plus facilement que les femmes une seconde union. Ainsi dans la génération 1950, 80,8\% des hommes de 60 ans pourraient vivre en couple contre $72,5 \%$ des femmes, seuls respectivement $74,4 \%$ et $68,1 \%$ étant mariés. Cette inégalité en défaveur des femmes est compensée par leur plus faible mortalité et, pour les uns

6. Signe d'une sélection moins négative des hommes par rapport aux femmes dans les générations plus récentes de divorcés. 
TABLEAU 6 Incidence des unions de fait sur la durée de vie en couple en fonction du sexe dans les générations 1940 et 1950

\begin{tabular}{|l|c|c|c|c|}
\hline \multirow{2}{*}{} & \multicolumn{2}{|c|}{ Hommes } & \multicolumn{2}{c|}{ Femmes } \\
\cline { 2 - 5 } & 1940 & 1950 & 1940 & 1950 \\
\hline Proportion ayant connu entre 60 ans et leur décès \\
\hline le mariage & 80,6 & 74,4 & 70,6 & 68,1 \\
\hline la vie en couple & 84,5 & 80,8 & 73,5 & 72,5 \\
\hline Durée de vie à 60 ans (en années) & 16,5 & 15,9 & 12,1 & 12,6 \\
\hline dans le mariage & 17,4 & 17,5 & 13,1 & 14,1 \\
\hline en union & 6,4 & 7,5 & 16,4 & 17,0 \\
\hline hors mariage & 5,5 & 6,1 & 15,5 & 15,4 \\
\hline hors union & \multicolumn{5}{|l}{} \\
\hline Proportion de la durée de vie passée & 72,2 & 67,8 & 42,5 & 42,5 \\
\hline dans le mariage & 76,0 & 74,2 & 45,8 & 47,8 \\
\hline en union & \multicolumn{5}{|l}{} \\
\hline
\end{tabular}

comme pour les autres, la prise en compte des unions non mariées allonge la durée de vie en couple de l'ordre d'une année dans la génération 1940 et d'une année et demie dans la génération 1950. La durée de l'isolement conjugal s'en trouve réduite d'autant. Ainsi, dans la génération 1950, les hommes de 60 ans pourraient passer 17,5 ans en union (15,9 ans dans le mariage) et 6,1 ans sans partenaire, ces durées atteignant respectivement 14,1 et 15,4 ans pour les femmes.

\section{DISCUSSION}

Au fil des générations, les durées passées dans le mariage des hommes et des femmes de 60 ans se sont allongées et rapprochées. Toutefois, ce sont, pour l'essentiel, les générations passées et présentes d'aînés qui ont bénéficié de cet allongement de la durée de vie dans le mariage. Â partir de la génération 1930, les structures conjugales des hommes et des femmes continueront à se rapprocher mais les évolutions de leurs destins risquent d'être plus contrastées qu'autrefois : le temps passé dans le mariage devrait davantage s'allonger chez les femmes que chez les hommes, l'inverse valant pour l'isolement matrimonial. Autrement dit, si dans la génération 1930 les femmes vivaient 2,9 fois plus longtemps sans conjoint que les hommes (respectivement 16,2 et 5,6 ans), pour leurs consœurs nées 20 ans plus tard ce coefficient pourrait n'être que de 2,3 (soit 17 ans contre 7,5 ans). 
À l'avenir, pour une fraction croissante d'hommes et de femmes, la vie après 60 ans se déroulera entièrement dans le divorce. Dans la génération 1950, tel pourrait être le cas de $13 \%$ de la population âgée pour des durées de vie de près de 29 ans pour les femmes et de plus de 20 ans pour les hommes, l'écart entre ces durées de vie s'étant creusé au détriment des hommes. De plus les personnes âgées hésitant moins à divorcer, $4 \%$ supplémentaires pourraient aussi connaître cette situation pour des durées plus longues encore. Le même type de constat vaut pour les célibataires: une part croissante d'hommes et de femmes passeront leur vie après 60 ans dans cette situation matrimoniale (de l'ordre de $10 \%$ ) et pour des durées de vie comparables à celle des divorcés.

Or les tendances de la mortalité de ces populations montrent qu'elles sont caractérisées par une fragilité de plus en plus importante au fil des générations. Côté masculin en particulier, le rythme de baisse de la mortalité des célibataires, et plus encore des divorcés, a été beaucoup plus faible que celui des autres populations, tendances conformes à celles observées dans d'autres pays (Murphy et collab., 2007). On peut toutefois penser que les divorcés et célibataires de demain s'adapteront mieux à leur solitude que les veufs d'aujourd'hui. Le divorce est, en effet, un événement moins systématiquement subi que le veuvage et il intervient, de surcroît, plus précocement dans la vie, donnant plus fortement le loisir aux personnes de s'adapter à leur nouvelle situation d'isolé. Cette argumentation est encore plus valable pour les célibataires. En revanche, on sait que le divorce a des conséquences négatives sur les échanges intergénérationnels, les hommes divorcés étant particulièrement vulnérables durant la vieillesse compte tenu de la faiblesse des liens entretenus avec leurs enfants. De même, l'intensité des échanges entre les mères divorcées et leurs enfants est moins forte que lorsque la famille n'a pas connu de rupture (Furstenberg et collab., 1995; Pezzin et Shone, 1999). Toutefois, il n'est pas exclu que la solidarité intergénérationnelle puisse être d'une autre nature dans les futures générations de divorcés en particulier chez les hommes, car ils auront davantage participé à l'éducation de leurs enfants (en raison, notamment, de la diffusion de la garde partagée). On peut aussi s'interroger sur les conséquences du recul de l'âge d'entrée dans le veuvage sur l'adaptation à cet événement : avec l'avancée en âge, en effet, les incapacités sont plus lourdes (Colin et Coutton, 200o) et les aidants familiaux potentiels, eux-mêmes plus âgés, moins efficients.

Toutefois, la situation matrimoniale légale reflète de moins en moins la réalité conjugale. La prise en compte des situations conjugales de fait conduit à une diminution de la durée de l'isolement conjugal identique 
chez les hommes et les femmes. Ce déclin pourrait être d'une année et demie dans la génération 1950, soit quasiment autant chez les hommes et plus chez les femmes que la progression estimée de la durée passée hors mariage. Autrement dit, contrairement au passé, quel que soit le sexe, les années de vie gagnées dans le futur pourraient n'être que des années de vie en couple. Et ce d'autant plus que ces données minimisent certainement la diffusion de l'union libre car elles ne permettent pas de saisir les nouvelles formes de conjugalité qui vont de pair avec le maintien de deux domiciles (Caradec, 1996). Toutefois, la prise en compte de ces données creuserait certainement les contrastes de situations entre les hommes et les femmes, l'isolement matrimonial des femmes étant beaucoup plus souvent synonyme de réelle absence de compagnon.

On peut, toutefois, se demander quelle est la nature de la solidarité entre conjoints au sein de ces nouvelles formes d'union. On sait par exemple que, d'une façon générale, les remariages, y compris côté féminin, fragilisent les échanges intergénérationnels : en comparaison des veuves, les femmes remariées reçoivent moins d'aide informelle et l'intensité des relations avec les enfants non biologiques est moins forte (Pezzin et Shone, 1999). En outre, revers de la médaille de cette prolongation de la durée de vie en couple, de plus en plus d'hommes seront confrontés à la dépendance de leur partenaire. Or les hommes d'aujourd'hui ont plus de difficultés à assumer la dépendance de leur épouse que l'inverse : ils ont plus souvent recours à des aides professionnelles (Martel et Légaré, 2001) ou placent plus fréquemment leur conjointe en institution (Delbès et collab., 2006). Ces évolutions risquent d'avoir des conséquences importantes sur le coût collectif de la prise en charge de la dépendance. Dans ce domaine toutefois, l'évolution de l'état de santé des futures générations de personnes âgées sera déterminante. Même si certaines modifications structurelles de la population âgée (élévation du niveau d'éducation, moindre pénibilité des professions) ou changements de comportements (attitude de prévention, meilleure alimentation et hygiène de vie) peuvent laisser penser que les personnes âgées de demain seront en meilleure santé que celles d'aujourd'hui, aucune tendance claire ne se dégageant du passé (Robine et collab., 2003), l'incertitude reste de rigueur sur les futurs changements de l'état de santé. 


\section{BIBLIOGRAPHIE}

Angel, R. J., J. L. Angel et C. L. Himes. 1992. « Minority group status, health transitions, and community living arrangements among the elderly ", Research on Aging, 14, $4:$ 496-521.

Arber, S., G. N. Gilber et M. Evandrou. 1988. « Gender, household composition and receipt of domiciliary services by elderly disabled people », Journal of Social Policy, $17: 153-175$.

Attias-Donfut, C. (dir.). 1995. Les solidarités entre les générations. coll. Essais et Recherches. Paris, Nathan, 352 p.

Bacon, B. et S. Pennec. 2007. APPSIM - Modelling Family Formation and dissolution. NATSEM/Canberra, 30 p. (NATSEM Online Working Paper, WP 4).

Barre, C. et M. VAnderschelden. 2004. L'enquête "Étude de l'histoire familiale » de 1999 - Résultats détaillés. Insee Résultats, 33, 47 p.

Breuil-Genier, P. 1998. "La dépendance des personnes âgées : recours aux proches et aux aides professionnelles », dans France : Portrait Social, INSEE : 91-107.

Bouffard, N., R. Easther, T. Johnson, R. J. Morrison et J. Vink. 2001. « Matchmaker, Matchmaker, Make me a match », Brazilian Electronic Journal of Economics, 4, 22.

Caradec, V. 1996. "Les formes de la vie conjugale des “jeunes" couples "âgés” ", Population, 4-5: 897-928.

Cassan, F., M. Mazuy et F. Clanché. 2001. "Refaire sa vie de couple est plus fréquent pour les hommes ", INSEE Première, 797, juillet.

Chappell, N. L. 1991. "Living arrangements and sources of care giving ", Journal of Gerontology, 46 : S1-S8.

Colin, C. et V. Coutton. 2000. "Le nombre de personnes âgées dépendantes d'après l'enquête Handicaps-incapacités-dépendance ", DRESS, Études et Résultats, 94, 7 p.

Cooney, T. et P. Uhlenberg. 1990. "The role of divorce in men's relations with adult children after mid-life », Journal of marriage and the family, 52 : 677-88.

Daguet, F. 1995. Un siècle de démographie française : structure et évolution de la population de 1901 à 1993. Paris, INSEE, INSEE Démographie-société, 47-48, 306 p.

DAGUET, F. 2002. Un siècle de fécondité française : caractéristiques et évolution de la fécondité de 1901 à 1999. INSEE, INSEE Résultats-Société, 8, 305 p.

David, M.-G. et C. STARZEC. 1996. " Aisance à 60 ans, dépendance et isolement à 80 ans ", INSEE Première, 447, avril.

De Jong Gierveld, J., T. Van Tilburg et L. Lecchini. 1997. «Socio-economic resources, household composition and social network as determinants of wellbeing among Dutch and Tuscan older adults », Genus, III, 3-4: 75-100.

Delbès, C. et J. Gaymu. 2003a. La retraite, 15 ans après. Paris, Ined, Les Cahiers de l'Ined, 154, $223 \mathrm{p}$. 
Delbès, C. et J. Gaymu. 2003b. « Passé 60 ans, de plus en plus souvent en couple? » Population et Sociétés, 389, 4 p.

Delbès, C. et J. Gaymu. 2004. "L'histoire conjugale des 50 ans et plus », dans C. Lefèvre et A. Filhon (dir.), Histoires de familles, histoires familiales, Paris, Ined, Les Cahiers de l'Ined, $156: 339-356$.

Delbès, C., J. Gaymu et S. Springer. 2006. «Les femmes vieillissent seules et les hommes vieillissent à deux. Un bilan européen ", Population et Sociétés, 419, $4 \mathrm{p}$.

Desesquelles, A. et N. Brouard. 2003. "Le réseau familial des personnes âgées de 60 ans et plus vivant à domicile ou en institution ", Population, 2 : 200-227.

Dolinsky, A. L. et I. Rosenwaike. 1988. "The role of demographic factors in the institutionalization of the elderly ", Research on Aging, 10, 2 : 235-257.

Duée, M., C. Rebillard et S. Pennec. 2005. "Les personnes dépendantes en France : Évolution et Prise en charge », IUSSP international population conference, Tours (France), 18-24 juillet.

Ettner, S. L. 1995. "The impact of "parent care" on female labor supply decisions ", Demography, 32, $1:$ 63-80.

Furstenberg, F. Jr., S.D. Hoffman et L. Shrestha. 1995. « The effect of divorce on intergenerational transfers : New evidence ", Demography, 32, $3:$ 319-332.

Glaser, K., M. Murphy et E. Grundy. 1997. "Limiting long-term illness and household structure among people aged 45 and over, Great Britain 1991 », Ageing and Society, $17:$ 3-19.

Grundy, E. et K. Glaser. 1997. " Trends in, and transitions to, institutional residence among older people in England and Wales 1971-1991 ", Journal of Epidemiology and Community Health, 51 : 531-540.

Grundy, E. 2006. "Ageing and vulnerable people : European perspectives », Ageing and Society, $26: 105-134$.

Hammel, E., A., K. W. Wachter et C. K. McDaniel. 1981. "The kin of the aged in 2000 ", dans J. Morgan, V. Oppenheimer et S. Kiesler, Social Change, vol. 2, New York, Academic Press : 11-39.

Hammel, E. A, C. Mason et K. W. Wachter. 1990. SOCSIM II, A sociodemographic Microsimulation Program, Rev. 1.o., Operating Manual, 29: 76- (5).

Hyrenius, H. et I. Adolfsson. 1964. A fertility simulation model. Göteborg, Almqvist \& Wiksell (distr.), 31 p.

Kalogirou, S. et M. Murphy. 2006. "Marital status of people aged 75 and over in nine EU countries in the period 2000-2030 », European Journal of Ageing, 3 : 74-81.

Le Bras, H. 1973. " Parents, grands-parents, bisaïeux », Population, 28, 1: 9-38.

Leridon, H. 1977. Human fertility: the basic components. Chicago, University of Chicago Press, 202 p.

Manzoli, L., P. Villari, G. M. Pirone et A. Boccia. 2007. " Marital status and mortality in the elderly : a systematic review and meta-analysis ", Social science and medicine, $64: 77-94$. 
Martel, L. et J. LÉGARÉ. 2001. «Avec ou sans famille proche à la vieillesse : une description du réseau de soutien informel des personnes âgées selon la présence du conjoint et des enfants », Cahiers québécois de démographie, 30, 1: 89-114.

Murphy, M., E. Grundy et S. Kalogirou. 2007. « The increase in marital status differences in mortality up to the oldest age in seven European countries, 1990-99 ", Population Studies, 61, 3:287-298.

Orcutt, G. H., M. Greenberger, J. Korbel et A. M. Rivlin.1961. Microanalysis of socioeconomic systems; a simulation study. New York, Harper, $425 \mathrm{p}$.

Pennec, S. 1996. «La place des familles à quatre générations en France », Population, 51, $1: 31-60$.

Perese, K. 2002. "Mate matching for microsimulation models ", Technical Paper 3. Washington (DC), Congressional Budget Office.

Pezzin, L. E. et B. S. Shone. 1999. "Parental marital disruption and intergenerational transfers : an analysis of lone elderly parents and their children », Demography, 36, $3: 287-297$.

Pickard, L., R. Wittenberg, A. Comas-Herrera, B. Davies et R. Darton. 2000. "Relying on informal care in the new century? Informal care for elderly people in England to 2031 ", Ageing and Society, $20: 745-772$.

RICCI, M. 1991. « Residential care for the elderly in Italy : recent trends and regional differences », European Population Conference, Paris, 21-25 octobre.

Robine, J.-M., I. Romieu et J.-P. Michel. 2003. «Trends in health expectancies », dans J.-M. Robine, C. Jagger, C. D. Mathers, E. M. Crimmins et R. M. Suzman (dir.), Determining health expectancies, Chichester (West Sussex), Wiley : 75-101.

Ruggles, S. 1987. Prolonged connections: The rise of the extended family in nineteenthcentury England and America. Madison/London, The University of Wisconsin Press, 283 p.

Schulz, R., A. T. O’Brien, J. Bookwala et K. Fleissner. 1995. «Psychiatric and physical morbidity effects of dementia caregiving : prevalence, correlates and causes ", The Gerontologist, 35, $6: 771-791$.

Sheps, M. C., J. A. Menken et A. P. Radick. 1973. Mathematical models of conception and birth. Chicago, University of Chicago Press, $428 \mathrm{p}$.

Smith, J. E et J. Oeppen. 1993. " "Estimating numbers of kin" in historical England using demographic microsimulation ", dans D. S. ReHer et D. SCHofield (dir.), Old and New Methods in Historical Demography, Oxford, Clarendon Press.

Spiess, C.K. et A.U. Schneider. 2003. "Interactions between care-giving and paid work hours among European midlife women, 1994 to 1996 », Ageing and Society, 23 : 41-68.

Soldo, B. J., D. A. Wolf et E. M. Agree. 1990. «Family, households, and care arrangements of frail older women : a structural analysis ", Journal of Geronto$\log y, 45:$ S238-249. 
Tomassini, C. et D. A. Wolf. 2000. "Shrinking Kin Networks in Italy Due to Sustained Low Low Fertility ", European Journal of Population, 16, 4:353-372.

United Nations. 2005. Living arrangements of older persons around the world. New York: Department of Economic and Social Affairs, Population division, United Nations, 138 p.

Vallin, J. et F. Meslé. 2001. Tables de mortalité françaises pour les XIX et XX $X^{e}$ siècles et projections pour le XXI ${ }^{e}$ siècle. Ined, Données statistiques 4, 102 p. + CD rom.

Vallin, J. et F. Meslé. 2002. "Comment améliorer la précision des tables de mortalité aux grands âges? », Population, 57, 4-5: 603-632.

Van Imhoff, E. et N. W. KeILman. 1991. LIPRO 2.o: An application of a dynamic demographic projection model to household structure in The Netherlands, NIDI CBGS Publications 23. Amsterdam/Lisse : Swets \& Zeitlinger.

Van Imhoff, E. et W. Post. 1997. "Méthodes de micro-simulation pour des projections de population ", Population, 52, 4:889-932.

Walker, A., J. Alber et A. M. Guillemard. 1993. Older People in Europe, Social and Economic Policies. The 1993 Report of the European Observatory, Commission of the European Communities, Directorate General V, Employment, Social Affairs, Industrial Relations, $36 \mathrm{p}$.

Wilson, B. et S. SMALLwood 2008. "The proportion of marriages ending in divorce », Population Trends, $131:$ 28-36. 


\section{ANNEXE MÉTHODOLOGIQUE}

Les raisons de l'utilisation d'un modèle dynamique de microsimulation ayant été déjà mentionnées dans le texte, cette annexe s'attache à décrire le modèle et les hypothèses retenues. Comme dans tout modèle de microsimulation dynamique, le point de départ est une population constituée d'enregistrements individuels, ici en l'occurrence de personnes âgées de 60 ans et plus appartenant aux générations 1895 à 1955 . Cette population vieillit de cet âge jusqu'au décès au gré de la survenue des événements simulés, à savoir la mortalité, la nuptialité, la divortialité et le veuvage et, pour les générations 1935 et 1955, la formation et la dissolution des unions non mariées.

\section{Population de départ}

Le choix de la population de départ dépend du type de simulation retenue. On peut décider de simuler la biographie de chaque personne de sa naissance au décès et s'intéresser ensuite uniquement à la période après 60 ans ou alors simuler seulement la biographie de 60 ans au décès. Simuler le cycle de vie entier a pour avantage de pouvoir déterminer la durée dans l'état matrimonial à tout âge mais requiert de prendre en compte l'ensemble des événements possibles pour obtenir la bonne répartition de la population par âge. Ainsi, il serait nécessaire de simuler les migrations par âge et sexe, données difficiles à obtenir en France sur la période actuelle et plus encore pour le passé. Le même constat de difficulté d'obtention de données d'enquêtes fiables et détaillées vaut pour la prise en compte de l'instabilité croissante des unions aux jeunes âges. L'exactitude de la structure matrimoniale initiale, c'est-à-dire celle réellement observée à 60 ans, a donc été privilégiée.

Comme il s'agit d'un modèle par cohorte, la population de départ peut donc soit être créée ${ }^{7}$, soit reposer sur des données réelles. L'avantage de la seconde solution est de pouvoir projeter des effectifs réels de population, et tel a été notre choix en raison, notamment, de notre souhait d'intégrer, à terme, des taux d'incapacité et d'évaluer des effectifs de population dépendante. Un échantillon représentatif de la population de chaque génération a été retenu, échantillon créé à partir des estimations de populations

7. Par exemple, grâce à un nombre identique de personnes de chaque sexe et état matrimonial à la date initiale de la simulation ou un nombre identique pour chaque génération avec une répartition par état matrimonial correspondant à la structure observée pour la génération 
de l'Insee par âge, sexe et état matrimonial légal (Daguet, 1995 et tableaux Population T6 sur le site de l'INSEE). L'échantillon est constitué de $3 \%$ de la population âgée de 60 ans, soit environ 1089200 personnes, les effectifs variant entre environ 8500 pour la génération la moins nombreuse (1917) et près de 25000 pour la plus nombreuse (1951). Les dernières données disponibles étant celles de 2005, les populations à 60 ans sont observées jusqu'à la génération 1945. Pour les générations plus récentes (1945 à 1955), la dernière population disponible en 2005 a été utilisée (soit par exemple celle observée à 50 ans pour la génération 1955) et la simulation a donc débuté pour elles avant l'âge de 60 ans.

\section{Ordre de simulation des événements}

Dans un modèle de microsimulation dynamique, des probabilités sont appliquées à chacun des individus de la population afin de déterminer s'il connaîtra ou non les divers événements simulés, et ce, en fonction de ses caractéristiques. Par exemple, pour chaque laps de temps, on teste si une personne reste ou non en vie, se marie ou non, etc. Compte tenu du nombre d'événements simulés et des données disponibles, il est impossible de calculer des taux qui prennent en compte tous les événements simultanément. Chaque événement est simulé l'un après l'autre, ce qui repose sur une hypothèse d'une certaine indépendance entre les événements (pas d'événements concurrents). Cette indépendance est relative et dépend de l'ordre dans lequel sont simulés les événements. L'ordre de simulation n'est, bien sûr, pas neutre : par exemple, si la mortalité est intégrée en premier, une personne sélectionnée pour décéder ne pourra divorcer ou se marier. Il doit donc suivre un ordre logique, c'est pourquoi la probabilité de mourir par âge a été appliquée en premier afin d'évaluer ceux qui vont survivre une année de plus. Ensuite, on a affecté aux survivants mariés la probabilité de devenir veuf et, sinon, celle de divorcer ou non, puis aux survivants célibataires celle de se marier ou non. Enfin, la probabilité de se remarier a été appliquée aux divorcés ou nouvellement divorcés et aux veufs ou nouvellement veufs.

La mortalité et le veuvage ont été simulés avant le divorce et le mariage car l'on peut supposer que ceux qui vont s'engager dans une nouvelle union ou se désengager sont moins susceptibles de mourir dans l'année que ceux qui n'ont pas de projets conjugaux. Le divorce et le veuvage sont simulés avant le mariage afin que les personnes ayant récemment subi une rupture d'union puissent se remarier dans l'année, probabilité forte pour au moins un des partenaires en cas de divorce. On a également supposé 
que les nouveaux mariages duraient au moins une année, autrement dit qu'ils ne pouvaient être rompus par le veuvage et le divorce durant leur première année.

Compte tenu de cet ordre des événements, la surmortalité des veufs durant la première année de leur veuvage n'a pu être prise en compte, ce qui a certainement pour conséquence de gonfler le nombre de ceux qui décèdent mariés.

Enfin, la période de simulation a été choisie suffisamment courte (le mois), afin de réduire, voire pratiquement éliminer, toute erreur liée à la non-prise en compte des événements concurrents.

\section{Sources de données et hypothèses relatives aux calculs des taux}

Les principales sources utilisées sont les statistiques d'état civil par âge, sexe et état matrimonial et les estimations de populations réalisées par l'INSEE (Daguet, 2002). Ces données présentent quelques incohérences, en particulier, la répartition de la population est tantôt disponible par classe d'âge tantôt par année d'âge, certaines données/années étant même parfois manquantes, surtout aux grands âges. Dans un premier temps, il a donc fallu harmoniser les séries. De manière générale, les valeurs disponibles ont été utilisées pour évaluer les données manquantes. Ainsi, les nondéclarations ont été réintégrées en faisant l'hypothèse d'une équirépartition dans la population considérée. Les données par classes d'âge ont été réparties en années d'âge à partir des distributions disponibles les années les plus proches.

En matière de mortalité, les données manquantes, en particulier certaines années de guerre, ont été comblées grâce aux estimations faites par Vallin et Meslé (2001, 2002). Jusqu'en 1942, les décès de veufs et divorcés ne faisaient qu'une catégorie. Les deux séries ont été séparées à partir des répartitions observées en 1943-1945 et en tenant compte d'un indicateur de surmortalité des divorcés. Après 90 ans, compte tenu de la faiblesse des effectifs des décès dans certains états matrimoniaux, la mortalité différentielle n'a pu être prise en compte et passé 100 ans les risques de décès calculés par Vallin et Meslé (2001) ont été utilisés. L'âge maximum au décès a été fixé à 105 ans, le risque étant alors de 1.

Compte tenu de la rareté des mariages et divorces après 80 ans, cet âge a été retenu comme seuil limite de survenue de ces événements.

Le veuvage est ici la conséquence du décès du conjoint et dépend, pour l'essentiel, de l'écart d'âge entre eux. Afin de déterminer l'âge du conjoint, les personnes mariées à 60 ans ont été, dans un premier temps, dissociées 
selon qu'elles étaient ou non dans un premier mariage. L'on sait en effet que la différence d'âge est plus importante, particulièrement côté masculin, lorsqu'il s'agit d'un second mariage (Delbès et Gaymu 2004). Pour chaque sexe, des distributions de différences d'âge selon l'âge à l'union et le type d'union (premier mariage ou remariage) ont ensuite été appliquées.

En outre, pour les générations 1935 et suivantes, les probabilités pour les célibataires, veufs et divorcés de vivre en couple ont été estimées en fonction du sexe et de l'état matrimonial. Les femmes non mariées ont, en effet, de plus faibles chances que les hommes de vivre en couple et les divorcés de plus fortes que les veufs ou les célibataires (Cassan, Mazuy et Clanché, 2001). Dans le modèle, une imputation des personnes non mariées est effectuée selon l'âge et la situation matrimoniale légale. Les personnes mariées sont toutes considérées comme vivant en couple. Comme précédemment pour les unions légales, des distributions d'écarts d'âges entre partenaires selon l'âge à la mise en union et l'état matrimonial ont été prises en compte pour déterminer les différences d'âge entre conjoints non mariés. Sur tous ces aspects des unions non mariées, les données sont issues de l'enquête "Étude de l'histoire familiale » de 1999 (Barre et Vanderschelden, 2004). Compte tenu de l'absence de données sur la mortalité des personnes non mariées et vivant en couple, la mortalité des mariés leur a été appliquée.

Une fois les données harmonisées par année d'âge, sexe et situation conjugale, les taux pour chaque événement ont été calculés, taux transversaux qui ont, ensuite, été transformés en taux par générations. Les taux calculés ou estimés couvrent la période 1900 à 2065, leur transformation en taux longitudinaux permettant de suivre les générations nées entre 1895 et 1955.

\section{Hypothèses d'évolutions futures}

\section{Mortalité et veuvage}

Après 2005, des disparités de mortalité selon l'état matrimonial ont été intégrées aux risques de décéder estimés par Vallin et Meslé : les écarts moyens observés durant la période 1990-2004 ont été prolongés tendanciellement (voir figure 1). En raison de la faiblesse des effectifs aux âges élevés, certaines valeurs visiblement trop (ou pas assez) élevées ont été modifiées afin obtenir des séries de taux plus cohérentes selon l'âge. En raison de cette fragilité des données, passé un certain âge ( 85 ans), la prise en compte d'une mortalité différentielle s'est avérée impossible. 
Le veuvage étant le résultat du décès du conjoint, il évolue au cours du temps en fonction à la fois de la mortalité et de l'écart d'âge entre conjoints, ce dernier variant au fil des générations.

\section{Mariage, divorce, union de fait}

Les tendances observées durant les dix dernières années ont été prolongées sur la période 2006-2015 (voir les tableaux 1 et 2 pour les évaluations), les probabilités de se marier ou de divorcer ayant, ensuite, été maintenues constantes aux niveaux atteints en 2015. L'augmentation de la proportion de personnes en seconde union est supposée se poursuivre à l'avenir et cette évolution est prise en compte au fil des générations.

\section{Formation et dissolution des unions non mariées}

Les taux de mise en union de fait sont basés sur les taux de nuptialité projetés affectés d'un coefficient multiplicateur. Ce coefficient, calculé sur la base des données disponibles dans l'enquête «Étude de l'histoire familiale » de 1999, diffère selon le sexe, le groupe d'âge et la situation matrimoniale légale.

Pour la dissolution de ces unions, faute de données plus précises, les taux utilisés sont identiques aux taux de divorce.

\section{Lissage des indices}

Même si les données utilisées sont issues de l'état civil, elles se sont parfois avérées très erratiques, notamment aux très grands âges pour les périodes anciennes. Les taux par générations ont donc été lissés grâce au package $\mathrm{R}$ de lissage de R. Hyndman (http://robjhyndman.com/publications/ software). Les quotients de mortalité et de veuvage sont lissés en utilisant des régressions splines pondérées avec des contraintes d'évolution monotone (weighted penalised regression splines with monotonicity constraints). Des régressions quadratiques pondérées localement (weighted locally quadratic regressions) sont utilisées pour le lissage des taux de nuptialité et de divortialité. 


\begin{abstract}
Sophie PENNEC and Joëlle GAYMU

Time spent in unpartnered and partnered states among old people in France: What has changed for men and women over time?

The aim of this article is to examine the trends in time spent in unpartnered states (years spent single, widowed or divorced), since these are periods of higher vulnerability for older people, especially in case of disability. We use a microsimulation model to analyse the changes in life expectancies by marital status for French people aged 60 and over, born between 1900 and 1950. Over all the cohorts, time spent in unions has increased for both men and women, and the sex gap has diminished. Past and present cohorts of older people have gained the most in terms of this increased time spent as part of a couple. From the 1930 and later cohorts, time spent in unions should increase more for women than for men, with the reverse effect prevailing for time spent in unpartnered states.
\end{abstract}

